\title{
Angiogenesis related genes NOS3, CD14, MMP3 and IL4R are associated to VEGF gene expression and circulating levels in healthy adults
}

Abdelsalam Saleh', Maria G. Stathopoulou', Sébastien Dadé ${ }^{1}$, Ndeye Coumba Ndiaye ${ }^{1}$, Mohsen Azimi-Nezhad ${ }^{1,2}$, Helena Murray ${ }^{3}$, Christine Masson ${ }^{1}$, John Lamont ${ }^{3}$, Peter Fitzgerald ${ }^{3}$ and Sophie Visvikis-Siest ${ }^{1,4^{*}}$

\begin{abstract}
Background: Vascular endothelial growth factor (VEGF) plays a key role in angiogenesis. The aim was to assess the genetic connections between the angiogenesis-related NOS3, CD14, MMP3, IL4R, IL4 genes and VEGF expression and plasma levels.

Methods: The associations between VEGF plasma levels with the polymorphisms of NOS3, CD14, MMP3, IL4R, and IL4 were assessed in 403 healthy unrelated adults. The epistatic and environmental interactions were explored, including four VEGF-related polymorphisms previously identified. The VEGF expression in peripheral blood mononuclear cells was quantified $(n=65)$ for the VEGF121, VEGF145, VEGF165, and VEGF189 isoforms.

Results: The polymorphism rs1799983 of NOS3 was associated with the sum of all VEGF isoforms mRNA levels $(P=0.032)$ and VEGF145 ( $P=0.033)$. Rs1800779 of NOS3 interacted with rs3918226 of the same gene and with the rs 2569190 of CD14 ( $P=0.022, P=0.042$, respectively) for VEGF plasma levels. Other epistatic interactions included the rs 1801275 of $I L 4 R$ with the rs6921438 (VEGF-related variant) and rs3025058 of MMP3 ( $P=0.042, P=0.010$ respectively) and the rs2569190 of CD14 with the rs3025058 of MMP3 ( $P=0.0119)$. We also identified an interaction of rs 1800779 with obesity, high-density lipoprotein cholesterol and triglycerides $(P=0.018, P=0.005, P=0.043$, respectively) as well as the interaction of rs6921438 with hypertension $(P=0.028)$.

Conclusions: Our findings indicated that genetic variants of NOS3, CD14, MMP3 and IL4R are implicated in the determination of VEGF expression and plasma levels. Thus, they support the hypothesis that in physiological conditions there are complex biological relationships between pathways (such as angiogenesis and inflammation), which are involved in the development of chronic diseases.
\end{abstract}

Keywords: VEGF, NOS3, CD14, MMP3, IL4R

\section{Background}

Angiogenesis is the procedure of development of new vessels from the existing vasculature. One of the most potent angiogenesis regulators is the vascular endothelial growth factor A (VEGF-A or more commonly known as VEGF). VEGF is a highly conserved, disulfide-bonded dimeric glycoprotein of $34-46 \mathrm{kDa}$. It is generated by several cell

\footnotetext{
* Correspondence: Sophie.Visvikis-Siest@inserm.fr

Abdelsalam Saleh and Maria G. Stathopoulou are equal first authors.

'UMR INSERM U 1122, IGE-PCV "Interactions Gène-Environnement en

Physiopathologie Cardio Vasculaire", Université de Lorraine, Nancy F-54000,

France

${ }^{4}$ Geriatric Service, University Hospital of Nancy, Nancy, France

Full list of author information is available at the end of the article
}

types including neutrophils, macrophages, fibroblasts, peripheral blood mononuclear cells (PBMCs) and endothelial cells. Elevated circulating VEGF levels have been associated with several types of cancer and other chronic diseases including cardiovascular diseases (ischemic heart disease, heart failure, stroke), diabetes, immune and inflammatory disorders [1-3].

Other regulators of angiogenesis include the nitric oxide synthase $\left(\mathrm{NOS}_{3}\right)$, the $\mathrm{CD} 14^{+}$monocytes, the matrix metalloproteinases (MMPs), and the interleukin 4 (IL4).

NOS3 provides continuous local production of nitric oxide (NO). $\mathrm{NO}$ is an important angiogenesis mediator and/or effector also involved in endothelial function and 
thereby can influence vascular tonicity, insulin resistance, development of atherosclerosis and type 2 diabetes $[4,5]$. The increase in NO production via up regulation of $\mathrm{NOS}_{3}$ by VEGF indicates that the angiogenic effect of VEGF seems to be mediated by NO [6-8]

CD $14^{+}$monocytes are abundant in human peripheral blood and have strong potential to differentiate into endothelial cells [9]. CD14 is a multifunctional receptor and contributes to different biological and pathophysiological processes including apoptosis, sepsis, inflammatory diseases, angiogenesis and tumor growth. Therefore, CD14 has been demonstrated to be a candidate for developing anti-cancer medications in tumor growth and angiogenesis [10].

The MMPs are zinc-dependent proteinases with an ability to degrade enzymes [11]. They are involved in pathologic conditions characterized by excessive degradation of extracellular matrix, such as tumor metastasis, and arteriosclerosis [12]. Several pieces of evidence indicate associations between MMPs and VEGF and a link of MMPs with angiogenesis [12-18].

The IL4 is a glycoprotein secreted by activated T lymphocytes, basophils and mast cells [19]. Previous studies have indicated the involvement of IL4 in angiogenesis [20,21].

These molecules are implicated in a variety of biological procedures in both health and disease. The precise mechanisms involving these molecules in angiogenesis in different conditions are under investigation. However, due to their complexity, interpreting the origin of their biological connections is challenging. Therefore, their evaluation in the healthy state is believed to provide important information concerning the physiological relationships between these molecules before the development of a specific disease. For this reason, the current study was performed in a supposed healthy population, aiming to examine possible genetic links between angiogenesis related (NOS3, CD14, $M M P 3$, and $I L 4$ ) candidate genes and VEGF plasma levels and gene expression in the healthy state. This assessment could provide evidence of functionally angiogenesis-related polymorphisms. Furthermore, gene-gene (epistatic) and gene-environment interactions effects on VEGF plasma levels were also investigated. Those interaction analyses included 4 polymorphisms significantly associated with VEGF plasma levels in a previous genome-wide association study (GWAS), and explaining up to $50 \%$ of its inter-individual variability [3]. In silico structural analyses have been performed for the functional validation of the results.

\section{Methods}

\section{Study population}

The STANISLAS Family Study is a 10-year longitudinal survey involving 1,006 families from Vandoeuvre-lès-
Nancy, France between 1993-1995 [22]. Exclusion criteria included the presence of chronic disorders (cardiovascular diseases or cancer) and the personal history of cardiovascular disease. The study protocol was approved by the Local Ethics Committee of Nancy and all subjects gave written informed consent. Four hundred and three unrelated adults collected during the second examination of the STANISLAS Family Study were selected based on complete availability of data.

\section{Data collection and biological measurements}

The procedures of data collection have been previously described [22, 23]. Blood samples were collected after overnight fasting. Sodium EDTA-plasma was separated by centrifugation at $2000 \mathrm{~g}$ for $15 \mathrm{~min}$ at $4{ }^{\circ} \mathrm{C}$ and stored at $-196{ }^{\circ} \mathrm{C}$ in liquid nitrogen until analysis. VEGF plasma levels were quantified by Randox Ltd (Crumlin, UK) using a biochip array analyser (Evidence ${ }^{\circ}$ ) [24]. Total cholesterol (TC) was measured using a cholesterol oxidase-paraaminophenazone method, triglycerides using a glycerophosphate oxidase/paraaminophenazone alanylglycine glycine method, and high-density lipoprotein cholesterol (HDL-C) levels using a phosphotungstate method, while low-density lipoprotein cholesterol (LDL-C) levels were calculated using the Friedewald formula [25].

Hypertension was defined as systolic blood pressure $\geq 140 \mathrm{~mm} / \mathrm{Hg}$ or diastolic blood pressure $\geq 90 \mathrm{~mm} /$ $\mathrm{Hg}$. Body mass index (BMI) was calculated as weight (kilograms) divided by height (meters) squared. Obesity was defined as BMI $\geq 30 \mathrm{~kg} / \mathrm{m}^{2}$. Smokers were identified based on current smoking status.

\section{SNP selection}

The single nucleotide polymorphisms (SNPs) rs2569190, rs2243250, rs2010963, rs3918226, rs1799983, rs1800779, rs3025058, rs1805015 and rs1801275 of the NOS3, CD14, MMP3, IL4R, and IL4 genes (Table 1) were selected on the basis of their functionality on the related genes.

The SNPs rs6921438, rs4416670, rs6993770, and rs10738760, previously shown to be associated with VEGF levels [3], were used for the testing of epistatic effects.

\section{DNA isolation and genotyping}

The genomic DNA was isolated using the salting out method [26]. The polymorphisms rs2569190, rs2243250, rs2010963, rs3918226, rs1799983, rs1800779, rs3025058, rs1805015 and rs1801275 of the NOS3, CD14, MMP3, ILAR and IL4 genes were genotyped using multilocus genotyping assays previously described [27, 28]. The VEGF-related SNPs were genotyped by Genoscreen (http://genoscreen.fr), using a Sequenom iPLEX Gold assay-Medium Throughput Genotyping Technology [29]. 
Table 1 Characteristics of the studied polymorphisms

\begin{tabular}{|c|c|c|c|c|c|c|}
\hline Chromosome & SNPS & Minor allele & Common allele & Closed to/on gene & Minor allele Frequency & HWE ${ }^{a} P$ \\
\hline 5 & rs2569190 & G & A & CD14 molecule & 0.48 & 0.8332 \\
\hline 5 & rs 2243250 & $\mathrm{~T}$ & C & IL4 interleukin 4 & 0.15 & 0.6865 \\
\hline 6 & rs4416670 & C & C & MGC45491 and MRPL14 (near VEGF) & 0.47 & 0.5489 \\
\hline 6 & rs2010963 & C & G & VEGFA & 0.35 & 0.3243 \\
\hline 6 & rs6921438 & A & G & MGC45491 and MRPL14 (near VEGF) & 0.4 & 1 \\
\hline 7 & rs3918226 & T & C & NOS3 nitric oxide synthase 3 & 0.09 & 0.3379 \\
\hline 7 & rs1799983 & $\mathrm{T}$ & G & NOS3 nitric oxide synthase 3 & 0.35 & 0.7309 \\
\hline 7 & rs1800779 & G & A & NOS3 nitric oxide synthase 3 & 0.4 & 0.6624 \\
\hline 8 & rs6993770 & $\mathrm{T}$ & A & ZFPM2 & 0.28 & 0.9019 \\
\hline 9 & rs10738760 & G & A & VLDLR and KCNV2 & 0.48 & 0.9205 \\
\hline 11 & rs3025058 & - & $\mathrm{T}$ & MMP3 matrix metallopeptidase 3 & 0.48 & 1 \\
\hline 16 & rs1805015 & C & T & IL4R interleukin 4 receptor & 0.13 & 0.3821 \\
\hline 16 & rs1801275 & G & A & IL $4 \mathrm{R}$ interleukin 4 receptor & 0.18 & 0.8632 \\
\hline
\end{tabular}

${ }^{a}$ Hardy Weinberg Equilibrium

\section{Gene expression assays}

Total RNA was extracted from PBMCs in a subsample of 65 subjects with a MagNaPure automate, using the MagNA Pure LC RNA HP isolation kit and RNA HP Blood External lysis protocol [Roche Diagnostics, France]. Reverse transcription of total RNAs were performed using 200 units of M-MuLV Reverse Transcriptase with $0.25 \mu \mathrm{g}$ of oligos (dT) (Promega, France) as previously described [30]. Quantification of the transcripts coding for the VEGF isoforms (VEGF 121 , $\mathrm{VEGF}_{145}, \mathrm{VEGF}_{165}, \mathrm{VEGF}_{189}$ ), the beta 2 microglobulin $(\beta 2 \mathrm{M})$ control gene were performed using TaqMan ${ }^{\bullet}$ and LightCycler technologies (LC TaqMan Master Kit, Roche Diagnostics, France) in duplicate. RT-PCR optimization and specificity of Real Time-PCR products were conducted using SYBR $^{\odot}$ Green technology (LC FastStart DNA MasterPLUS SYBR Green I kit, Roche Diagnostic, France), melting curves analysis and agarose gel electrophoresis of the PCR amplicons [30]. All mRNA levels were normalized to the mRNA levels of $\beta 2 \mathrm{M}$ gene. Total VEGF mRNA derived from the sum of the ratio of the four isoforms present in PBMCs.

\section{Computational structural analyses}

The polymorphism rs1799983 allows the generation of two isoforms of endothelial NOS3: eNOS3 Glu298 (allele G) and eNOS3 Asp298 (allele T). This gene is located on chromosome 7 q36.1. It contains 26 coding exons extending on approximately $24 \mathrm{~kb}$. Multiple transcript variants encode different isoforms (Fig. 1). Canonical isoform codes for an enzyme of 1203 amino acids. The active protein is a homodimer (chains A and B) catalyzing from L-arginine the formation of NO. The polymorphism rs1799983 is located on the coding exon 7 of NOS3 and has been previously identified by Yoshimura et al. as a Glu298-to-Asp variant [31].

Sofowora et al. found that eNOS3 Asp298 homozygotes excreted significantly less nitrate/nitrite than eNOS3 Glu298 homozygotes [32]. As amino acid in position 298 does not belong to the catalytic residue of the enzyme active site (formed by $\operatorname{Arg187}(\mathrm{A})$ Cys184 (A) - Glu361 (A) - Trp356 (A)) a question remains: how this $\mathrm{rs} 1799983 \mathrm{G}>\mathrm{T}$ can affect nitric oxide production and how can this be linked with VEGF?

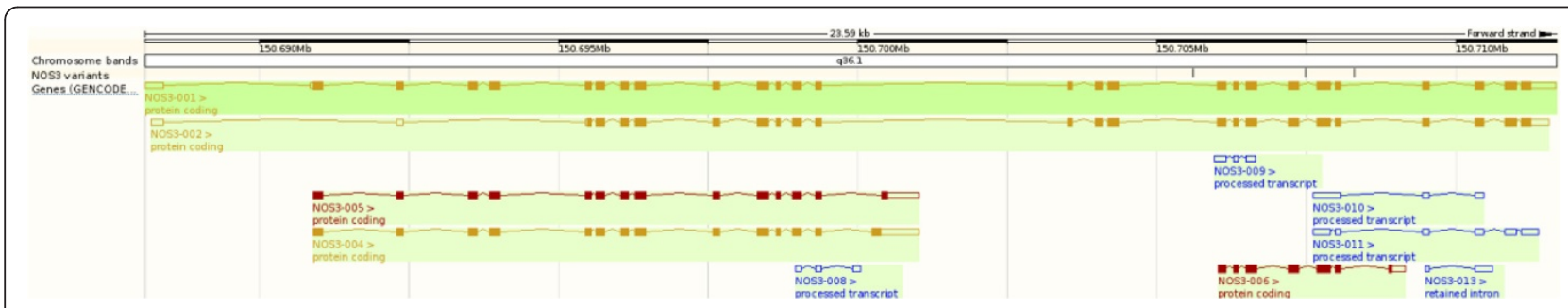

Fig. 1 Schematic organisation of eNOS3 transcripts in chromosome 7. Rs1799983 is indicated with the orange triangle. Exons are represented by rectangular shapes and coding exons are coloured 
Structural analyses were performed using the crystallographic structure of the human endothelial nitric oxide synthase, oxygenase domain [33]. Conformational changes in nitric oxide synthases induced by chlorzoxazone and nitroindazoles were assessed through crystallographic and computational analyses of inhibitor potency [33]. Computational analyses for the building of a model of NOS3 Asp298 were performed using the I-Tasser suite [34, 35] and Pymol software was used to analyze structural features (the PyMol Molecular Graphics System, Schrodinger, LLC).

\section{Statistical analyses}

VEGF plasma concentrations and mRNA values were log-transformed, before the analyses, to normalize their distribution. Hardy-Weinberg equilibrium was tested using the chi-square test.

The associations between the SNPs of NOS3, CD14, $M M P 3, I L 4 R$ and IL4 genes and VEGF plasma and expression levels were assessed through linear regression adjusted for age, gender and BMI under an additive model and using the minor allele as reference allele.

The epistatic interactions between the polymorphisms of NOS3, CD14, MMP3, IL4R and IL4 genes and the VEGFrelated SNPs for VEGF plasma levels were tested using the linear models described before with the introduction of the interaction term of a $2 \times 2$ combination between the assessed SNPs. For example VEGF $\sim$ age + gender + BMI + SNP1 + SNP2 + SNP1 $\times$ SNP2. The environmental factors used for the SNPs $\times$ environment interactions included smoking, blood lipids levels, obesity, and hypertension. We used linear regression models adjusted for age, gender, BMI (when obesity was the assessed factor, adjustments were performed only for age and gender), the environmental factor, and the additional interaction term (SNP $\times$ environmental factors). The interaction analyses were not performed for $V E G F$ gene expression data due to the limited sample size. Analyses were performed using PLINK 1.07 (http://pngu.mgh.harvard.edu/purcell/plink) [36] and the SPSS 17.0 (SPSS, Inc, Chicago, Illinois) statistical softwares. Significance was determined at a two-tailed $P=0.05$ level.

The sample size calculation was performed using the software QUANTO. Based on published information about allele frequencies of the selected polymorphisms, the levels of VEGF in plasma and its expression, and an estimation of a mild effect size, a number of 400 individuals would be sufficient in order to achieve a statistical power of $80 \%$.

\section{Results}

The studied polymorphisms are presented in Table 1. All SNPs were in agreement with Hardy-Weinberg equilibrium $(P>0.05)$. The characteristics of the studied population are summarized in Table 2 (Additional file 1: Tables S1 and S2).
Table 2 Characteristics of study population $(n=403)$

\begin{tabular}{lll}
\hline Variable & Mean $^{\mathrm{a}}$ & $\mathrm{SD}^{\mathrm{b}}$ \\
\hline Age (years) & 44.4 & 4.8 \\
Gender (\%) male & 50.4 & \\
Body mass index $\left(\mathrm{kg} / \mathrm{m}^{2}\right)$ & 24.9 & 3.9 \\
Vascular endothelial growth factor $(\mathrm{pg} / \mathrm{ml})$ & 42.7 & 43.3 \\
Total cholesterol $(\mathrm{mmol} / \mathrm{l})$ & 5.7 & 1.0 \\
Triglycerides (mmol/l) & 1.3 & 1.2 \\
High- density lipoprotein $(\mathrm{mmol} / \mathrm{l})$ & 1.6 & 0.4 \\
Low-density lipoprotein $(\mathrm{mmol} / \mathrm{l})$ & 3.5 & 0.8 \\
Obesity (\%) & $7.9 \%$ & \\
Smoking (\%) & $25.4 \%$ & \\
Hypertension (\%) & $5.7 \%$ & \\
\hline
\end{tabular}

${ }^{\mathrm{a}}$ Mean values for continuous variables and percentages for categorical variables

${ }^{\text {b }} \mathrm{SD}$ standard deviation

Association of the assessed polymorphisms of the NOS3, CD14, MMP3, IL4R and IL4 genes with VEGF gene expression and plasma levels

After adjustment for age, gender and BMI, a significant association was observed between rs1799983 of NOS3 and the sum of all VEGF isoforms mRNA and VEGF 145 mRNA $(\beta=0.19, P=0.032$ and $\beta=0.17, P=0.033$ respectively). None of the polymorphisms of the NOS3, CD14, $M M P 3, I L 4 R$ and IL4 genes were associated with VEGF plasma levels.

Epistatic interactions between NOS3, CD14, MMP3, IL4R, IL4 and VEGF-related polymorphisms on the plasma levels of VEGF

Six genetic variants were involved in epistatic interactions for VEGF plasma levels regulation, including rs1800779 and rs3918226 (NOS3), rs2569190 (CD14), rs3025058 (MMP3), $\quad$ rs1801275 (IL4R) and rs6921438 (VEGF) (Table 3). Specifically, the interaction of rs1800779 of NOS3 with rs3918226 of the same gene was associated with increased VEGF plasma levels $(\beta=0.17, P=0.022)$ and the interaction with the rs2569190 of CD14 $(\beta=-0.06$, $P=0.042$ ) was associated with decreased VEGF plasma levels. Therefore, the SNP rs1800779 of NOS3 has a modifiable effect on VEGF plasma levels though epistatic interactions with different polymorphisms. Additionally the

Table 3 Epistatic interactions for VEGF plasma levels

\begin{tabular}{llccl}
\hline SNP $\times$ SNP & Genes & $\beta^{\mathrm{a}}(\mathrm{pg} / \mathrm{ml})$ & $t$-values & $P$ \\
\hline rs1800779 $\times$ rs2569190 & NOS3 $\times$ CD14 & -0.07 & -2.04 & 0.042 \\
rs1800779 $\times$ rs3918226 & NOS3 $\times$ NOS3 & +0.17 & 2.29 & 0.022 \\
rs1801275 $\times$ rs6921438 & IL4R $\times$ VEGF & +0.08 & 2.043 & 0.042 \\
rs1801275 $\times$ rs3025058 & IL4R $\times$ MMP3 & -0.10 & -2.58 & 0.010 \\
rs2569190 $\times$ rs3025058 & CD $14 \times$ MMP3 & +0.07 & 2.36 & 0.019 \\
\hline
\end{tabular}

${ }^{a} \beta$, effect size 
rs1801275 of IL4R interacted with the VEGF-related SNP rs6921438 resulting in an increase of VEGF plasma levels $(\beta=0.07, P=0.042)$ and with the rs3025058 of MMP3 with a decreasing effect $(\beta=-0.09, P=0.010)$. Again, in this case, the rs1801275 has different effects on VEGF plasma levels depending on interactions with different polymorphisms of angiogenesis-related genes. Finally, the interaction between rs2569190 of CD14 and rs3025058 of $M M P 3$ was associated with a significant increase in plasma levels of VEGF ( $\beta=0.07, P=0.019)$.

\section{SNPs $\times$ environmental interactions on the plasma levels of VEGF}

Significant interactions were observed between NOS3 SNPs and HDL-C, triglycerides and obesity (Table 4). A decrease of VEGF plasma levels resulted from the interaction of rs 1800779 with obesity and triglycerides ( $\beta=-0.11, P=0.018, \beta=-0.04, P=0.043$ respectively), while its interaction with HDL-C was associated with an increase in VEGF plasma levels $(\beta=0.12, P=$ 0.005). Furthermore, the VEGF-related polymorphism rs6921438 interacted with hypertension to decrease VEGF levels $(\beta=-0.18, P=0.028)$.

\section{Structural analyses of NOS3 gene}

The study of NOS3 domains revealed that the amino acid in position 298 is located in NOS-Interacting Protein (NOSIP) binding domain (amino acids 98-498). Moreover, this binding domain overlaps the oxygenasedomain containing active site (Fig. 2).

Comparative analyses of the crystal structure of the human eNOS3 oxygenase domain (pubmedcode :1M9M -1.96 A resolution - Figs. 3 and 4) with the computed eNOS3 Asp298 reveals conformational changes between eNOS3 Glu298 and eNOS3 Asp298. Indeed, the amino acid in position 298 is not buried but exposed on the surface. Thereby, eNOS3 shows a protuberance when it is composed of Glu298 whereas eNOS3 model removes this relief with aspartate amino acid. (Figs. 5 and 6).

\section{Discussion}

In the present study, we have identified significant associations between genetic variants of NOS3 with VEGF gene expression in a healthy population. Furthermore, significant epistatic and gene $\times$ environment interactions have been identified for VEGF plasma levels involving polymorphisms of NOS3, CD14, MMP3, IL4R and VEGF-related SNPs.

Numerous SNPs have been identified in NOS3 gene, among them, the common variant, G984T (rs1799983). It is located in exon 7 of the gene, and it modifies its coding sequence, thus resulting in an amino acidic substitution of glutamic acid to aspartic acid at position 298 of the protein. It has been reported to alter its enzymatic activity [37, 38]. This SNP has been demonstrated by several groups to be linked with the risk for coronary spasm, coronary artery disease and acute myocardial infarction [39-43].

In this study, a significant association between the SNP rs1799983 and VEGF expression level (total mRNA and VEGF $_{145}$ isoform) was observed. The minor allele of this SNP was associated with increased levels of VEGF mRNA. Such association might suggest the existence of transcriptional regulation of VEGF from NOS3 in PBMCs in nonpathological situations and thus a novel molecular link between VEGF and NO. It is noteworthy that in a previous investigation, we had identified significant associations between the $\mathrm{VEGF}_{145}$ isoform with cellular adhesion and inflammation molecules expression (ICAM-1, L-selectin and TNF-a) in the same population [44]. Our current results further support the importance of this isoform on cardiovascular physiology and underline its potent role as a key molecule in different diseases.

There are few pathways leading to the synthesis of NO. One of them consists in the formation of the complex eNOS3/caveolin-1. However, a protein avidly binding to the eNOS oxygenase domain termed NOSIP [45] has been identified. When NOSIP binds to eNOS3, eNOS is sequestered in the Golgi apparatus decreasing NO production in eNOS3 Asp298 homozygotes [32]. It is therefore likely that eNOS3 298 Asp promotes formation of a complex with NOSIP. In this context, homozygotes eNOS3 298 Asp probably synthesize $\mathrm{NO}$ via alternative pathways involving VEGF/PI3K/Akt [46]. Thus, to compensate this engagement to this preferred pathway of complex formation with NOSIP for homozygotes eNOS3 Asp298, the physiological response to $\mathrm{NO}$ production is the increase of VEGF expression levels, known to stimulate the synthesis of $\mathrm{NO}$ via eNOS3 on the one hand but also via VEGF/PI3K/Akt.

Table 4 Significant gene $x$ environment interactions with VEGF plasma levels

\begin{tabular}{|c|c|c|c|c|}
\hline Interaction & Gene & $\beta^{a}(\mathrm{pg} / \mathrm{ml})$ & $t$-values & $P$ \\
\hline rs1800779 $\times$ HDL-C & NOS3 & 0.12 & 2.78 & 0.005 \\
\hline rs1800779 $\times$ Triglycerides & NOS3 & -0.04 & -1.98 & 0.043 \\
\hline rs1800779 $\times$ Obesity & NOS3 & -0.11 & -2.36 & 0.018 \\
\hline rs6921438 $\times$ Hypertention & MGC45491 and MRPL14(near VEGF) & -0.18 & -2.20 & 0.028 \\
\hline
\end{tabular}

${ }^{a} \beta$, effect size 


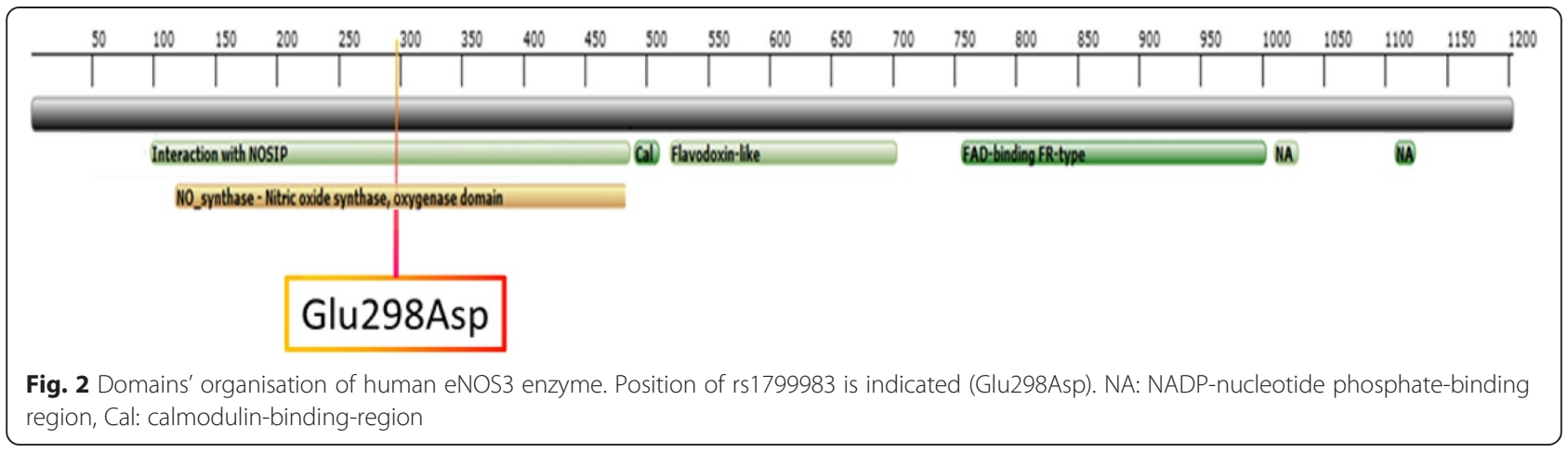

Therefore, our structural analyses offer an explanation of the link between the SNP rs1799983 and VEGF expression level.

On the other hand, no significant relations were found between $V E G F$ gene expression and/or plasma levels and CD14, MMP3, IL4R and IL4 polymorphisms in our healthy population.

In this study, we also observed an epistatic interaction between NOS3 SNP rs1800779 and CD14 SNP rs2569190 which resulted in a significant decrease in VEGF plasma level. The rs1800779 is located in the upstream and promoter region of the gene and may eventually lead to reduced production and bioavailability of NO. Also, the CD14 SNP rs2569190 is situated at position -159 of the gene and has been suggested to be linked with increased expression and transcriptional activity of CD14 [47].

Additionally, we have found that the interaction between the NOS3 SNPs rs1800779 and rs3918226, was associated with a significant increase in VEGF plasma level. Similar to the former SNP, the rs3918226 is located in the promoter region of NOS3 gene. Although no functional studies have been reported so far for this SNP, few studies have identified a transcription-factor binding site for the ETS-family next to rs3918226, suggesting that rs3918226 can potentially modulate the expression of NOS3 [48].

It is important to mention that the epistatic interaction between IL4R SNP rs1801275 and the VEGF-related SNP rs6921438 was associated with a significant increase in VEGF plasma level in our healthy population. IL4 biological actions are mediated by its binding to the receptors IL4, which leads to the activation of intracellular signaling pathways [49]. The IL4R SNP rs1801275 is a substitution of Arg with Gln at the position 576 of the protein, resulting in an enhancement in the receptor signaling. In addition, the VEGF-related SNP rs6921438 is an intergenic variant that is located at $171 \mathrm{~kb}$ downstream of the VEGF gene on chromosome 6p21.1. In a previous GWAS, the minor allele of the polymorphism was associated with decreased VEGF levels [3]. The mechanism of this interaction is not known and as few data are available in the literature, the biological explication of this epistatic effect cannot be hypothesized.

Complex relationships have been observed between IL4 and VEGF in several diseases. IL4 has pro-angiogenic and pro-inflammatory properties in the lungs during exposure to chronic hypoxia, an effect dependent on the hypoxiamediated induction of the VEGF signaling pathway [50].

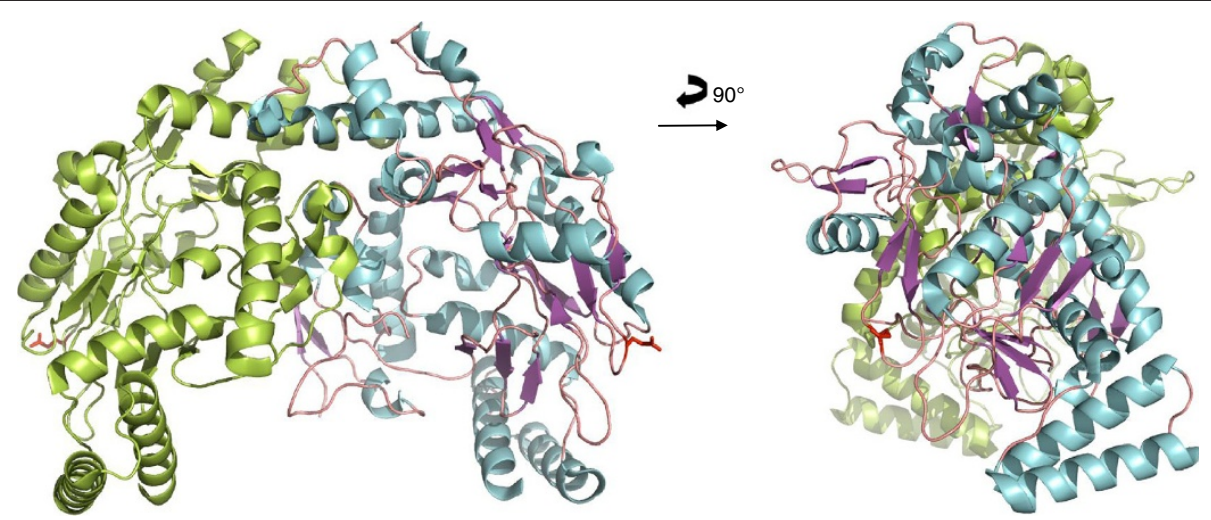

Fig. 3 Representation of the dimeric form of eNOS3 oxygenase domains (PDB : 1M9M). Chain A is in green and chain B is represented in cyan for alpha helix and magenta for beta strands. Glu298Asp corresponding to rs1799983, is depicted in red in each chain 


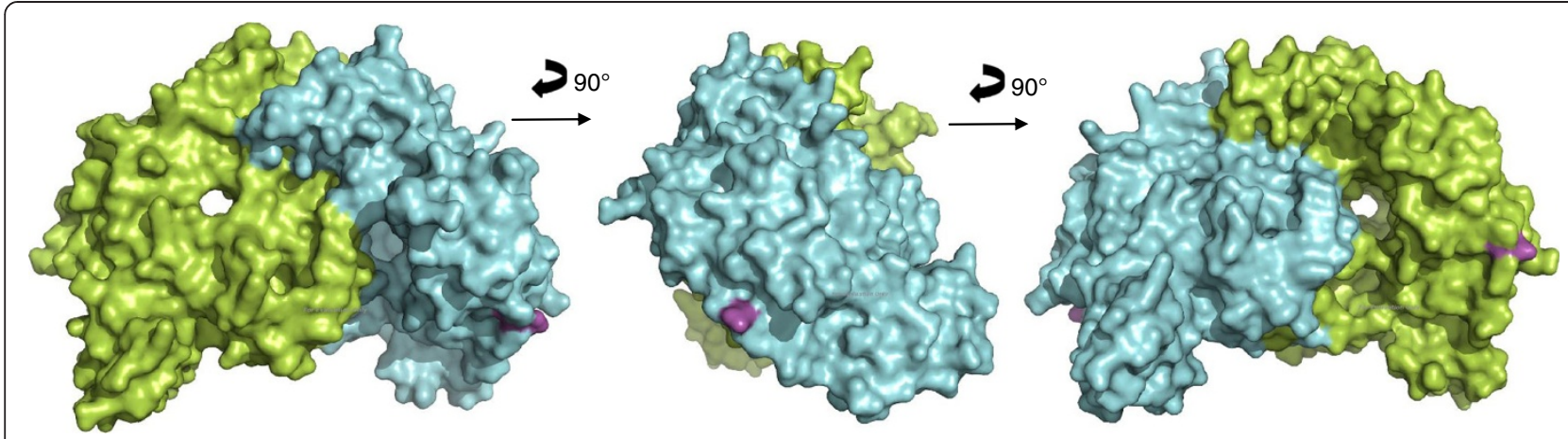

Fig. 4 Representation of solvent-accessible surface area of eNOS3 homodimer oxygenase domains (PDB :1M9M). Chain A and B are in green and cyan respectively. Glu298 is coloured in magenta in both chains

Also, IL4 has been discovered to exert anti-angiogenic properties in several cancer models with high VEGF expression levels [51]. Moreover, in patients with rheumatoid arthritis, IL4 inhibits VEGF production in synovial fibroblasts [7]. The results of our study support the concept of crosstalk between VEGF and IL4.

Another finding of our investigation indicates an epistatic interaction between the IL4R SNP rs1801275 and MMP3 SNP rs3025058 that was associated with a significant decrease in VEGF plasma level. The MMP3 SNP is located in the promoter region of the gene where the one allele has a run of six adenosines (6A) and another having five adenosines (5A) at position 1171 [52]. It has been shown that MMPs are involved in different steps of angiogenesis and play an important role in the blood vessel growth and the process of vascular development in cancer [53]. In addition, MMPs can increase the bioavailability of VEGF [54]. The association between MMPs and VEGF has been demonstrated, in several studies, to contribute to different pathological conditions, e.g. cancer invasion [15-17]. Therefore, considering the potential interactions of VEGF with both IL4 and MMPs, it is not surprising that in this study we observed a significant interaction between IL4R and MMPs for VEGF plasma levels.

Hypertension, smoking, blood lipids and obesity were the "environmental factors" selected for interaction analyses, as they are risk factors with large environmental impact for different diseases, especially cardiovascular diseases. We observed interactions between NOS3 SNP rs1800779 with HDL-C, triglycerides and obesity. These interactions were associated with increased VEGF plasma levels in the case of HDL-C, and decreased VEGF levels for triglycerides and obesity. Loebig et al. reported a positive correlation between plasma VEGF concentrations in overweight subjects as compared with normal and low weight individuals. However, they did not find any significant differences of VEGF levels when comparing normal and low weight participants [6]. Sandhofer et al., found that the plasma levels of VEGF are positively and negatively correlated with BMI in men, and visceral fat in women respectively [55]. Our interactions results indicate that the relationship between VEGF and adiposity could be mediated by NOS 3 variants, through interactions with the adiposity indexes.
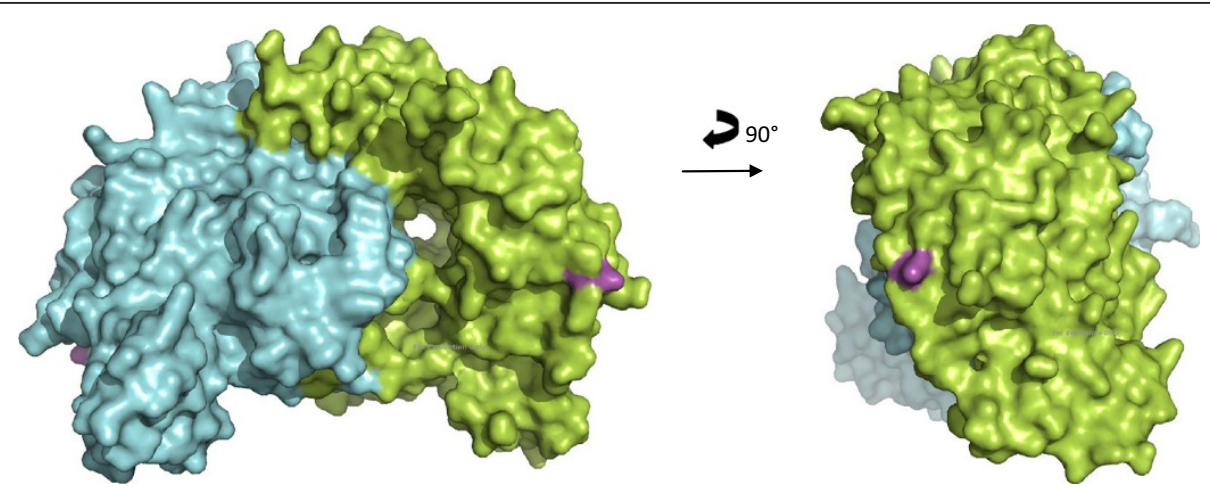

Fig. 5 NOS3 Glu298 [1m9m] 

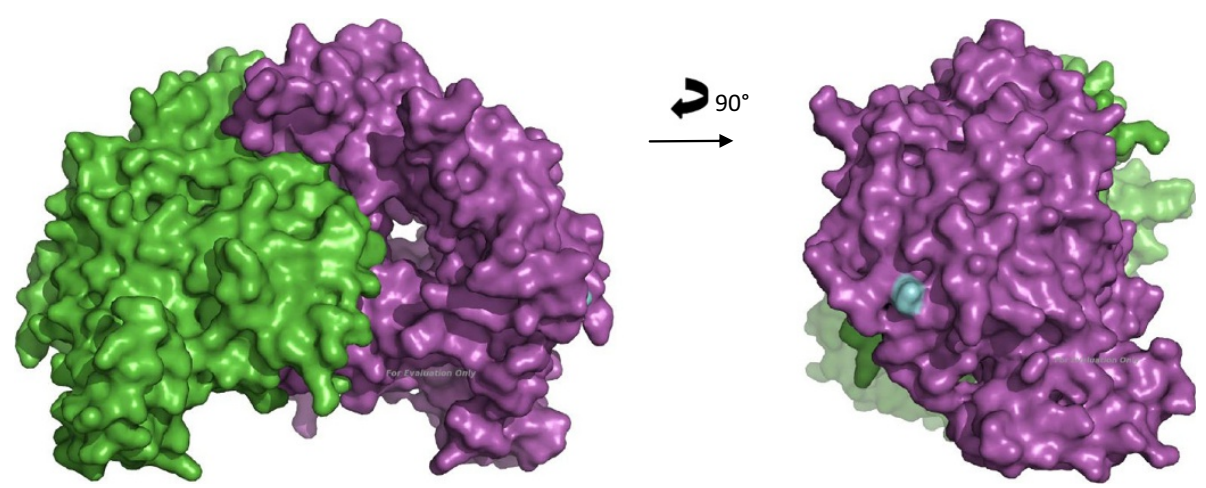

Fig. 6 Homodimer NOS3 Asp98 (cyan)

Finally, we identified an interaction of the SNP rs6921438 with hypertension, which was associated with a decrease in VEGF levels. As previously mentioned, the minor allele of this variant was associated with decreased VEGF levels [3] and explained $41 \%$ of the VEGF inter-individual variability. Furthermore, it was the only polymorphism among the 4 VEGF-related assessed in the present study that was implicated in epistatic and environmental interactions for VEGF. Therefore, it seems that its role as a VEGF biomarker may be even more significant through indirect interactions as well. Moreover, the same variant has been also recently found to contribute to decreased HDL-C and increased LDL-C levels in the same population [56]. Thus, it could represent a strong biological link between angiogenesis, blood pressure regulation and blood.

The findings of this study are original and they are providing initial evidences on the field of functional variants implicated in angiogenesis regulation, through direct effects or interactions, in health. A limitation of the study is the use of nominal significance threshold for the production of the results. This is the most common practice in the candidate genes studies, where a limited number of SNPs are assessed, like the present one. Understanding this limitation, we have performed structural analyses and provide functional biological evidences for some of these results. Furthermore, we acknowledge the need for replication of our results in larger population.

\section{Conclusions}

In the present work, we assessed the associations of VEGF plasma levels and gene expression with NOS3, CD14, MMP3, IL4R, IL4 and VEGF-related SNPs in a healthy population, as well as their epistatic and environmental interactions. The significant role of a functional NOS3 polymorphism on VEGF gene expression was presented and biological confirmation was provided though structural analyses of NOS3 gene. VEGF plasma levels were also affected by epistatic and environmental interactions with polymorphisms of the NOS3, CD14, $M M P 3$, and IL4R. Among the VEGF-related polymorphisms, the stronger VEGF determinant, rs6921438, was also implicated in such interactions modulating VEGF levels. These results indicate the central role of VEGF regulation in different physiological biological procedures and support the existence of complex relationships between angiogenesis, NOS3, CD14, MMP3 and IL4R that exist even in the healthy state. Such complex interactions should be taken into account in future studies concerning the implication of VEGF in related human chronic pathologies. The understanding of these associations may promote the knowledge of the molecular mechanisms and the processes that mediate complex diseases such as diabetes, cancer and cardiovascular diseases. Also the possible development of new therapeutic targets in a personalized medicine approach can also be considered. Furthermore, this study underlines the value of candidate gene approach in combination with GWAS in assessing interactions of the biological determinants of disease intermediate phenotypes such as the VEGF.

\section{Additional file}

Additional file 1: Table S1. VEGF plasma and expression levels per genotype. Table S2. VEGF plasma and expression levels per interaction condition. (DOCX $72 \mathrm{~kb}$ )

\section{Abbreviations}

VEGF: Vascular endothelial growth factor; NOS3: Nitric Oxide Synthase; NOSIP: NOS-Interacting Protein; CD14: Cluster of differentiation; MMP3: Matrix metalloproteinase-3; IL4: Interleukin-4; IL4R: Interleukin-4 receptor;

GWAS: Genome-wide association study; TC: Total cholesterol; HDL-C: Highdensity lipoprotein cholesterol; LDL-C: Low-density lipoprotein cholesterol; BMI: Body mass index; PBMC: Peripheral blood mononucleated cell; SNPs: Single-nucleotide polymorphism; ICAM: InterCellular Adhesion Molecule; TNFa: Tumor necrosis factor.

\section{Competing interests}

The authors declare that they have no competing interests. 


\section{Authors' contributions}

AS: study design, data interpretation, literature search, writing of the draft. MGS: study design, data analysis and interpretation, writing of the draft. SD: structural analyses, data interpretation and critical review of the manuscript. NCN: data interpretation and critical review of the manuscript. MAN: data interpretation, literature search, critical review of the manuscript. HM and CM: experiments and critical review of the manuscript. JL, PF: data interpretation, critical review of the manuscript. SVS: Conception and study design, data interpretation, critical review of the manuscript. All authors read and approved the final manuscript.

\section{Authors' information}

Not applicable.

\section{Acknowledgments}

Samples and data used for this study are part of the Biological Resources Center (BRC): "Interactions Gène-Environnement en Physiopathologie CardioVasculaire" (IGE-PCV) in Nancy, France.

\section{Funding}

This work was funded through the Collaborative Biolntelligence Program. The funding source had no implication in study design; in the collection, analysis and interpretation of data; in the writing of the report; and in the decision to submit the article for publication.

\section{Author details}

'UMR INSERM U 1122, IGE-PCV "Interactions Gène-Environnement en Physiopathologie Cardio Vasculaire", Université de Lorraine, Nancy F-54000, France. ${ }^{2}$ Department of Medical Genetics, School of Medicine, Mashhad University of Medical Sciences, Mashhad, Iran. ${ }^{3}$ Randox Laboratories, Crumlin, UK. ${ }^{4}$ Geriatric Service, University Hospital of Nancy, Nancy, France.

\section{Received: 21 October 2014 Accepted: 23 September 2015 Published online: 05 October 2015}

\section{References}

1. Kiriakidis S, Andreakos E, Monaco C, Foxwell B, Feldmann M, Paleolog E. VEGF expression in human macrophages is NF-kappaB-dependent: studies using adenoviruses expressing the endogenous NF-kappaB inhibitor IkappaBalpha and a kinase-defective form of the IkappaB kinase 2. J Cell Sci. 2003;116(Pt 4):665-74.

2. Blann AD, Belgore FM, McCollum CN, Silverman S, Lip PL, Lip GY. Vascular endothelial growth factor and its receptor, Flt-1, in the plasma of patients with coronary or peripheral atherosclerosis, or Type II diabetes. Clin Sci (Lond). 2002;102(2):187-94.

3. Debette $\mathrm{S}$, Visvikis-Siest $\mathrm{S}$, Chen $\mathrm{MH}$, Ndiaye NC, Song C, Destefano A, et al. Identification of cis- and trans-acting genetic variants explaining up to half the variation in circulating vascular endothelial growth factor levels. Circ Res. 2011;109(5):554-63.

4. Aicher A, Heeschen C, Mildner-Rihm C, Urbich C, Ihling C, Technau-Ihling K, et al. Essential role of endothelial nitric oxide synthase for mobilization of stem and progenitor cells. Nat Med. 2003;9(11):1370-6.

5. Podar K, Anderson KC. The pathophysiologic role of VEGF in hematologic malignancies: therapeutic implications. Blood. 2005;105(4):1383-95.

6. Loebig M, Klement J, Schmoller A, Betz S, Heuck N, Schweiger U, et al. Evidence for a relationship between VEGF and BMI independent of insulin sensitivity by glucose clamp procedure in a homogenous group healthy young men. PLoS ONE. 2010;5(9):e12610.

7. Hong KH, Cho ML, Min SY, Shin YJ, Yoo SA, Choi JJ, et al. Effect of interleukin-4 on vascular endothelial growth factor production in rheumatoid synovial fibroblasts. Clin Exp Immunol. 2007;147(3):573-9.

8. Isenberg JS, Martin-Manso G, Maxhimer JB, Roberts DD. Regulation of nitric oxide signalling by thrombospondin 1: implications for anti-angiogenic therapies. Nat Rev Cancer. 2009:9(3):182-94.

9. Fernandez Pujol B, Lucibello FC, Gehling UM, Lindemann K, Weidner N, Zuzarte ML, et al. Endothelial-like cells derived from human CD14 positive monocytes. Differentiation. 2000;65(5):287-300.

10. Song M-N, Cho S-Y. CD14 Acts as an Angiogenic Factor by Inducing Basic Fibroblast Growth Factor (bFGF). Bull Korean Chem Soc. 2007;28(9):1613-4.

11. Egeblad M, Werb Z. New functions for the matrix metalloproteinases in cancer progression. Nat Rev Cancer. 2002;2(3):161-74.
12. Westermarck J, Li S, Jaakkola P, Kallunki T, Grenman R, Kahari VM. Activation of fibroblast collagenase-1 expression by tumor cells of squamous cell carcinomas is mediated by p38 mitogen-activated protein kinase and c-Jun NH2-terminal kinase-2. Cancer Res. 2000;60(24):7156-62.

13. Itoh T, Tanioka M, Matsuda H, Nishimoto H, Yoshioka T, Suzuki R, et al. Experimental metastasis is suppressed in MMP-9-deficient mice. Clin Exp Metastasis. 1999;17(2):177-81.

14. Gatto C, Rieppi M, Borsotti P, Innocenti S, Ceruti R, Drudis T, et al. BAY 12-9566, a novel inhibitor of matrix metalloproteinases with antiangiogenic activity. Clin Cancer Res. 1999:5(11):3603-7.

15. Bergers G, Brekken R, McMahon G, Vu TH, Itoh T, Tamaki K, et al. Matrix metalloproteinase- 9 triggers the angiogenic switch during carcinogenesis. Nat Cell Biol. 2000;2(10):737-44.

16. Zhou Z, Apte SS, Soininen R, Cao R, Baaklini GY, Rauser RW, et al. Impaired endochondral ossification and angiogenesis in mice deficient in membranetype matrix metalloproteinase I. Proc Natl Acad Sci U S A. 2000;97(8):4052-7.

17. Chang C, Werb Z. The many faces of metalloproteases: cell growth, invasion, angiogenesis and metastasis. Trends Cell Biol. 2001;11(11):S37-43.

18. Silvestre JS, Mallat Z, Tamarat R, Duriez M, Tedgui A, Levy BI. Regulation of matrix metalloproteinase activity in ischemic tissue by interleukin-10: role in ischemia-induced angiogenesis. Circ Res. 2001;89(3):259-64.

19. Carr C, Aykent S, Kimack NM, Levine AD. Disulfide assignments in recombinant mouse and human interleukin 4. Biochemistry. 1991;30(6):1515-23.

20. Faffe DS, Flynt L, Bourgeois K, Panettieri Jr RA, Shore SA. Interleukin-13 and interleukin-4 induce vascular endothelial growth factor release from airway smooth muscle cells: role of vascular endothelial growth factor genotype. Am J Respir Cell Mol Biol. 2006;34(2):213-8.

21. Haas CS, Amin MA, Allen BB, Ruth JH, Haines 3rd GK, Woods JM, et al. Inhibition of angiogenesis by interleukin-4 gene therapy in rat adjuvantinduced arthritis. Arthritis Rheum. 2006;54(8):2402-14.

22. Visvikis-Siest S, Siest G. The STANISLAS Cohort: a 10-year follow-up of supposed healthy families. Gene-environment interactions, reference values and evaluation of biomarkers in prevention of cardiovascular diseases. Clin Chem Lab Med. 2008;46(6):733-47.

23. Siest G, Visvikis S, Herbeth B, Gueguen R, Vincent-Viry M, Sass C, et al. Objectives, design and recruitment of a familial and longitudinal cohort for studying gene-environment interactions in the field of cardiovascular risk: the Stanislas cohort. Clin Chem Lab Med. 1998;36(1):35-42.

24. Fitzgerald SP, Lamont JV, McConnell Rl, el Benchikh O. Development of a high-throughput automated analyzer using biochip array technology. Clin Chem. 2005;51(7):1165-76.

25. Friedewald WT, Levy RI, Fredrickson DS. Estimation of the concentration of low-density lipoprotein cholesterol in plasma, without use of the preparative ultracentrifuge. Clin Chem. 1972;18(6):499-502.

26. Miller SA, Dykes DD, Polesky HF. A simple salting out procedure for extracting DNA from human nucleated cells. Nucleic Acids Res. 1988;16(3):1215.

27. Cheng S, Pallaud C, Grow MA, Scharf SJ, Erlich HA, Klitz W, et al. A multilocus genotyping assay for cardiovascular disease. Clin Chem Lab Med. 1998;36(8):561-6.

28. Hoppe C, Klitz W, Cheng S, Apple R, Steiner L, Robles L, et al. Gene interactions and stroke risk in children with sickle cell anemia. Blood. 2004;103(6):2391-6.

29. Ehrich M, Bocker S, van den Boom D. Multiplexed discovery of sequence polymorphisms using base-specific cleavage and MALDI-TOF MS. Nucleic Acids Res. 2005;33(4):e38.

30. Marteau JB, Mohr S, Pfister M, Visvikis-Siest S. Collection and storage of human blood cells for mRNA expression profiling: a 15-month stability study. Clin Chem. 2005;51(7):1250-2.

31. Yoshimura M, Yasue $H$, Nakayama M, Shimasaki $Y$, Sumida H, Sugiyama $S$, et al. A missense Glu298Asp variant in the endothelial nitric oxide synthase gene is associated with coronary spasm in the Japanese. Hum Genet. 1998;103(1):65-9.

32. Sofowora G, Dishy $V$, Xie HG, Imamura H, Nishimi $Y$, Morales $C R$, et al. In-vivo effects of Glu298Asp endothelial nitric oxide synthase polymorphism. Pharmacogenetics. 2001;11(9):809-14.

33. Rosenfeld RJ, Garcin ED, Panda K, Andersson G, Aberg A, Wallace AV, et al. Conformational changes in nitric oxide synthases induced by chlorzoxazone and nitroindazoles: crystallographic and computational analyses of inhibitor potency. Biochemistry. 2002;41(47):13915-25. 
34. Zhang Y. I-TASSER server for protein $3 \mathrm{D}$ structure prediction. BMC Bioinformatics. 2008:9:40.

35. Roy A, Kucukural A, Zhang Y. I-TASSER: a unified platform for automated protein structure and function prediction. Nat Protoc. 2010;5(4):725-38.

36. Purcell S, Neale B, Todd-Brown K, Thomas L, Ferreira MA, Bender D, et al. PLINK: a tool set for whole-genome association and population-based linkage analyses. Am J Hum Genet. 2007;81(3):559-75.

37. Ryk C, Wiklund NP, Nyberg T, de Verdier PJ. Polymorphisms in nitric-oxide synthase 3 may influence the risk of urinary-bladder cancer. Nitric Oxide. 2011;25(3):338-43.

38. Venturelli E, Villa C, Fenoglio C, Clerici F, Marcone A, Ghidoni R, et al. The NOS3 G894T (Glu298Asp) polymorphism is a risk factor for frontotemporal lobar degeneration. Eur J Neurol. 2009;16(1):37-42.

39. Hibi K, Ishigami T, Tamura K, Mizushima S, Nyui N, Fujita T, et al. Endothelial nitric oxide synthase gene polymorphism and acute myocardial infarction. Hypertension. 1998;32(3):521-6.

40. Colombo MG, Andreassi MG, Paradossi U, Botto N, Manfredi S, Masetti S, et al. Evidence for association of a common variant of the endothelial nitric oxide synthase gene (Glu298 $\rightarrow$ Asp polymorphism) to the presence, extent, and severity of coronary artery disease. Heart. 2002;87(6):525-8.

41. McNamara DM, Holubkov R, Postava L, Ramani R, Janosko K, Mathier M, et al. Effect of the Asp298 variant of endothelial nitric oxide synthase on survival for patients with congestive heart failure. Circulation. 2003;107(12):1598-602.

42. Rios DL, D'Onofrio LO, Souza JK, Queiroz AM, Raduy-Maron L, Silva-Neto N et al. Smoking-dependent and haplotype-specific effects of endothelial nitric oxide synthase gene polymorphisms on angiographically assessed coronary artery disease in Caucasian- and African-Brazilians. Atherosclerosis. 2007;193(1):135-41.

43. Morray B, Goldenberg I, Moss AJ, Zareba W, Ryan D, McNitt S, et al. Polymorphisms in the paraoxonase and endothelial nitric oxide synthase genes and the risk of early-onset myocardial infarction. Am J Cardiol. 2007;99(8):1100-5.

44. Azimi-Nezhad M, Stathopoulou MG, Bonnefond A, Rancier M, Saleh A, Lamont J, et al. Associations of vascular endothelial growth factor (VEGF) with adhesion and inflammation molecules in a healthy population. Cytokine. 2013;61(2):602-7.

45. Dedio J, Konig P, Wohlfart P, Schroeder C, Kummer W, Muller-Esterl W. NOSIP, a novel modulator of endothelial nitric oxide synthase activity. FASEB J. 2001;15(1):79-89.

46. Zachary I. Signaling mechanisms mediating vascular protective actions of vascular endothelial growth factor. Am J Physiol Cell Physiol. 2001;280(6):C1375-86.

47. LeVan TD, Bloom JW, Bailey TJ, Karp CL, Halonen M, Martinez FD, et al. A common single nucleotide polymorphism in the CD14 promoter decreases the affinity of Sp protein binding and enhances transcriptional activity. J Immunol. 2001;167(10):5838-44.

48. Salvi E, Kutalik Z, Glorioso N, Benaglio P, Frau F, Kuznetsova T, et al. Genomewide association study using a high-density single nucleotide polymorphism array and case-control design identifies a novel essential hypertension susceptibility locus in the promoter region of endothelial NO synthase. Hypertension. 2012;59(2):248-55.

49. Roger T, Froidevaux C, Le Roy D, Reymond MK, Chanson AL, Mauri D, et al. Protection from lethal gram-negative bacterial sepsis by targeting Toll-like receptor 4. Proc Natl Acad Sci U S A. 2009;106(7):2348-52.

50. Yamaji-Kegan K, Su Q, Angelini DJ, Johns RA. IL-4 is proangiogenic in the lung under hypoxic conditions. J Immunol. 2009;182(9):5469-76.

51. Saleh M, Wiegmans A, Malone Q, Stylli SS, Kaye AH. Effect of in situ retroviral interleukin-4 transfer on established intracranial tumors. J Natl Cancer Inst. 1999;91(5):438-45.

52. Ye S, Eriksson P, Hamsten A, Kurkinen M, Humphries SE, Henney AM. Progression of coronary atherosclerosis is associated with a common genetic variant of the human stromelysin-1 promoter which results in reduced gene expression. J Biol Chem. 1996;271(22):13055-60

53. Nguyen M, Arkell J, Jackson CJ. Human endothelial gelatinases and angiogenesis. Int J Biochem Cell Biol. 2001;33(10):960-70.

54. Liutkeviciene R, Zaliaduonyte-Peksiene D, Zaliuniene D, Gustiene O, Jasinskas $V$, Lesauskaite $V$, et al. Does matrix metalloproteinase-3 polymorphism play a role in age-related macular degeneration in patients with myocardial infarction? Medicina (Kaunas). 2012;48(8):404-9.
55. Sandhofer A, Tatarczyk T, Kirchmair R, Iglseder B, Paulweber B, Patsch JR, et al. Are plasma VEGF and its soluble receptor sFlt-1 atherogenic risk factors? Cross-sectional data from the SAPHIR study. Atherosclerosis. 2009;206(1):265-9.

56. Stathopoulou MG, Bonnefond A, Ndiaye NC, Azimi-Nezhad M, El Shamieh S, Saleh $A$, et al. A common variant highly associated with plasma VEGFA levels also contributes to the variation of both LDL-C and HDL-C. J Lipid Res. 2013;54(2):535-41.

\section{Submit your next manuscript to BioMed Central and take full advantage of:}

- Convenient online submission

- Thorough peer review

- No space constraints or color figure charges

- Immediate publication on acceptance

- Inclusion in PubMed, CAS, Scopus and Google Scholar

- Research which is freely available for redistribution 